K. Beutel ${ }^{1}$

A. Simon ${ }^{2}$

\section{Diagnostik und Therapie Katheter-assoziierter} Infektionen in der pädiatrischen Onkologie

\author{
Diagnostic and Management of Central Venous Line Infections \\ in Pediatric Cancer Patients
}

\section{Zusammenfassung}

Bei jedem Patienten mit zentralvenösem Verweilkatheter (central venous access device, CVAD) muss bei klinischen Zeichen einer Infektion ohne weiteren Fokus immer von einer CVAD-assoziierten Infektion ausgegangen werden. Die Sicherung der Diagnose gelingt mit Abstrichen, Blutkulturen und ggf. Kultur der Katheterspitze oder des Portreservoirs. Häufigste Erreger sind grampositive Keime, insbesondere Koagulase-negative Staphylokokken (CoNS). Bei Verdacht auf Infektion bei pädiatrisch onkologischen Patienten ist vor der Explantation des Systems meistens der Versuch einer in-situ-Therapie über den CVAD gerechtfertigt (IB). Ein intraluminal infizierter CVAD sollte explantiert werden, wenn unter einer in vitro effektiven antibakteriellen Chemotherapie nach mehr als 72 Stunden weiterhin Erreger in der Blutkultur nachweisbar sind (IB) oder früher, falls sich Vitalzeichen und Allgemeinzustand des Patienten unter der insitu-Behandlung deutlich verschlechtern (IB). Die gesicherte Infektion des CVAD durch bestimmte Erregerspezies, wie z.B. S. aureus, Pseudomonas aeruginosa oder multiresistente Acinetobacter baumannii und Candida spp. erfordern die umgehende Entfernung des Systems, da die Heilungsraten niedrig und septische Komplikationen häufig sind (IB). Bei positiver Blutkultur sollte die antibakterielle Chemotherapie nach klinischer Genesung frühestens nach 7 Tagen, möglichst nach Rekonvaleszenz der neutrophilen Granulozyten und nach mindestens zwei sterilen Kontrollen der aus dem CVAD entnommenen Blutkultur beendet werden (IIC). Antibiotika-Heparin-Blocklösungen haben sich bei grampositiven Infektionen bewährt (IB). Über den adju-

\section{Abstract}

Otherwise unexplained clinical signs of infection in patients with tunneled or totally implanted central venous access devices (CVAD) are highly suspicious of an underlying CVAD-associated infection. Diagnostic methods include catheter swabs, blood cultures and cultures of the catheter tipp or port reservoir. In case of a suspected CVAD-related blood stream infection in pediatric cancer patients in situ treatment without prompt removal of the device can be tried. The removal of the CVAD should be considered, if bacteremia persists or relapses 72 hours or longer after the initiation of an (in vitro effective) antibacterial therapy administered through the line. The CVAD should be removed even earlier, if the patient suffers from hypotension or other signs of severe organ dysfunction related to the infection. If S. aureus, Pseudomonas aeruginosa, multiresistant Acinetobacter baumannii or Candida spp.are isolated from blood cultures taken through a CVAD, patients are at a high risk for severe complications and immediate device removal is also recommended. Duration of therapy depends on the immunological recovery of the patient (neutrophils counts), the pathogen isolated and on the presence of related complications like thrombosis, pneumonia, endocarditis, osteomyelitis. Antibiotic-lock techniques in addition to systemic treatment are beneficial in Gram-positve infections. Although prospectively controlled studies are missing, the concomitant use of urokinase- or taurolidine seems to favour catheter salvage. Pädiatrische Hämatologie und Onkologie am Universitätsklinikum Bonn am Universitätsklinikum · Adenauerallee $119 \cdot 53113$ Bonn · Tel.: +49/(0)2 28/2 873254 E-mail: asimon@ukb.uni-bonn.de 
vanten Einsatz von Urokinase oder Taurolidin in der Therapie kann zur Zeit nur individuell entschieden werden (IIB).

\section{Stichwörter}

Diagnose und Therapie - zentralvenöse Katheter · Kinder mit Krebserkrankung

\section{Key words}

Diagnosis and therapy · intravascular catheter · child · cancer

\section{Hintergrund}

In der Behandlung pädiatrisch-onkologischer Patienten werden seit etwa 20 Jahren dauerhafte zentralvenöse Katheter (CVAD, central venous access devices) verwendet. Dies sind entweder getunnelte und nahe der Eintrittsstelle mit einer Kunststoffmanschette (cuff) ausgestattete CVAD vom Typ Broviac, Hickman oder Groshong [16, 21]. Alternativ werden Port-Systeme mit einem subkutanen Reservoir implantiert [88, 131, 132]. Der Einsatz dauerhafter CVAD bietet für Patient und Behandlungsteam erhebliche Vorteile. CVAD dienen zur Verabreichung von gewebetoxischen Zytostatika, antimikrobieller Therapie, von Analgetika und von Blutprodukten. Sie erleichtern die Durchführung einer hyperosmolaren parenteralen Ernährung bei hochgradiger Mukositis [30]. Außerdem erlauben sie schmerzlose Blutentnahmen bei Kindern und Jugendlichen, die ohnehin durch die Erkrankung und Therapie erheblich belastet sind. Auch bei Kindern mit hämatologischen Erkrankungen, wie bei Kindern mit Thalassämie, erleichtern CVAD die Therapie [85]. Im Notfall sind sie stets als großlumiger Zugang für die akute Schockbehandlung verfügbar. Diese Übersicht bezieht sich ausschließlich auf dauerhaft implantierte CVAD. Nicht getunnelte, zentralvenöse Katheter werden in der pädiatrischen Onkologie nur als Übergangs- oder Notlösung im Rahmen einer besonders intensiven Behandlungsphase eingesetzt.

Die Implantation von Fremdmaterial in das Gefäßsystem erhöht das Risiko von Infektionen [84, 88, 94, 95, 100, 117, 145]. Hinzu kommen mechanisch bedingte Probleme (Dislokation, Rhythmusstörung, Katheterruptur, Paravasat, Chylothorax) [10, 102, 137] und thrombotische Komplikationen [54, 74, 96, 97].

In zwei aktuellen prospektiven Studien mit pädiatrisch onkologischen Patienten überwogen die mechanischen mit 4,5 (mit 1,3 ) vs. 1,7 (vs. 0,87) Ereignissen/1000 Anwendungstage (utilisation days, UD) die infektiösen Komplikationen [26, 54]. Hierbei ist stets zu beachten, ob als Bezugsgröße auch die ambulanten „Anwendungstage" in die Berechnung mit eingehen. Wenn dies geschieht, resultieren „günstige“ Ergebnisse unter 0,5 Infektionen pro 1000 UD. Nach einer prospektiven Studie CVAD-assoziierter Infektionen, die zwischen diesen beiden Möglichkeiten unterschied, war die Inzidenzdichte während des stationären Aufenthaltes um den Faktor 25 höher (7,4 vs. 0,3/1000 Anwendungstage) [132]. In der aktuellen Zwischenauswertung (Stand 01.04.2005) der Onkopäd NKI-Studie (prospektive Surveillance nosokomialer Infektionen in der pädiatrischen Onkologie, Studienleiter A. Simon) liegt die kumulative Inzidenzdichte für CVADassoziierte Bakteriämien für den Broviac bei 1,77 (0,9-2,41; insgesamt 18040 UD) und für den Port bei 1,82 (0,65-5,43; insgesamt 8264 UD). Naturgemäß ist das Risiko infektiöser Komplikationen während des intensiven Gebrauchs (stationäre Therapie mit vielen Manipulationen) am höchsten.
In dieser Übersicht sollen - nach einigen praxisbezogenen Hinweisen zum Erregerspektrum und zur Pathogenese - Grundprinzipien des Managements CVAD-assoziierter Infektionen bei pädiatrisch-onkologischen Patienten beschrieben werden ${ }^{1}$. Die hier vorgestellten Konsensusempfehlungen sollen den eigenverantwortlichen Entscheidungsprozess der behandelnden pädiatrischen Onkologinnen und Onkologen unterstützen [92, 110, $111,136]$.

\section{Erregerspezies}

Bei den im Zusammenhang mit Katheterinfektionen aus Abstrichen, zentralen Blutkulturen oder von explantiertem Kathetermaterial isolierten Bakterien handelt es sich in den meisten Fällen um grampositive Bakterien [148]. Überwiegend sind dies vom distalen Katheterende (Hub) $[124,125]$ oder von der Haut stammende, in der Mehrzahl Methicillin-resistente koagulasenegative Staphylokokken (CoNS bzw. MRSE) [147].

Bei Patienten mit Langzeit-parenteraler Ernährung konnte gezeigt werden, dass ein Teil der CoNS-Infektionen durch Translokation aus dem Darm stammt $[43,44]$.

Wegen der hohen Morbidität bedeutsam sind S. aureus (MRSA, falls Methicillin-resistent) [134]. Deutlich seltener finden sich Keime aus dem Darm (Enterokokken, VRE falls Vancomycin-resistent) [133, 135] oder dem Oropharynx [78] (vergrünende, $\alpha$-hämolysierende Streptokokken: Streptococcus viridans und Stomatococcus spp.) [57, 144, 148] als Auslöser von Katheterinfektionen. Meist mit einem septischen Schock gehen CVAD-Infektionen einher, die durch gramnegative Spezies [68] wie Pseudomonas aeruginosa, Burkholderia cepacia, Klebsiella spp., Stenotrophomonas maltophilia [55] oder Acinetobacter baumannii verursacht werden [31]. Zudem kommen CVAD-Infektionen durch Candida spp. vor ${ }^{2}$, oft wird in diesem Kontext C. parapsilosis genannt $[38,46,64,87,101,105,106]$.

Raritäten, die eine sofortige Entfernung des CVAD erfordern, sind Lokalinfektionen durch Aspergillus spp. [11] oder durch atypische Mykobakterien [109].

\footnotetext{
${ }^{1}$ Die spezielle antibakterielle Therapie bakterieller Infektionen bei pädiatrisch onkologischen Patienten wird im Beitrag von A. Simon in diesem Band diskutiert, während die Beiträge von H.J.Laws und von A. Groll die empirische Therapie bei Granulozytopenie-Fieber bzw. die gezielte Therapie bei Pilzinfektionen besprechen.

${ }^{2}$ Siehe Beitrag von A. Groll in diesem Band.
} 


\section{Für die Therapie relevante pathogenetische Faktoren}

Die mikrobielle Besiedlung des CVAD beginnt unmittelbar nach Implantation und erfolgt zunächst intraluminal, ausgehend von einer Kontamination des Katheterhub [125] oder von der Eintrittstelle (Punktionsstelle des Port) [79]. Viel seltener sind kontaminierte Infusionslösungen [90] oder eine sekundär hämatogene Besiedlung $[78,143]$. Die Kolonisation kann schon 24 Stunden nach Katheterimplantation nachgewiesen werden. Bakterien, die in der Lage sind, über extrazelluläre Glykopolysaccharide an mit Fibrin und Fibronektin benetzte Kunststoffe zu binden, stellen den Kern des Problems dar [147]. Die rasche Ausbildung einer extrazellulären Biofilmmatrix verhindert eine suffiziente Immunantwort gegen adhärierende Bakterien [36, 98, 99, 113]. Der Biofilm erschwert zudem die Keimeradikation durch eine in vitro hochwirksame antibakterielle Therapie [3, 4, 9, 34]. An der Aktivität gegen solche, an Kunststoff adhärente und in Biofilm eingebettete Bakterien muss sich eine Substanz messen lassen, wenn sie in der Prävention $[3,4,8]$ und Therapie von CVAD-Infektionen eingesetzt werden soll [63] Wirksam sein kann diese Therapie nur dann, wenn am Wirkort über einen ausreichend langen Zeitraum Konzentrationen des Antibiotikums vorliegen die mind. 100fach, besser 1000fach über der minimalen Hemmkonzentration des Erregers liegen [45, 127]. Zusätzlich wird versucht, die Integrität des Biofilms vor der Antibiotikagabe durch Urokinase [6, 8, 39] oder Ethanol [37] anzugreifen.

\section{Definition und Diagnose}

Lokale CVAD-assoziierte Infektionen gehen häufig mit lokalen Entzündungszeichen (Rötung, Schwellung, Schmerzen, Sekretion) an der Katheteraustrittsstelle oder im Katheterverlauf einher und stellen somit eine klinische Diagnose dar. Allerdings können die lokalen Symptome bei granulozytopenischen Patienten nur gering ausgeprägt sein. Fieber ist häufig das einzige Symptom von CVAD-assoziierten Bakteriämien. Das Auffiebern eines Patienten unmittelbar nach Spülung des oder nach Anschluss einer Infusion an den CVAD sollte unbedingt den Verdacht auf eine Besiedlung oder Infektion des Katheters lenken.

Dies trifft ohne Einschränkung auch für Patienten mit CVAD zu, die nicht granulozytopenisch sind $[2,60,123]$. Auch bei diesen Patienten sollte stets eine Blutkultur aus dem CVAD entnommen werden.

Als „CVAD-assoziierte Infektion“ gilt ein Ereignis, bei dem klinische Zeichen wie Fieber, Schüttelfrost, Erniedrigung des mittleren arteriellen Drucks, verminderte Durchblutung der Kreislaufperipherie, Oligurie mit oder ohne positive Blutkultur bei einem Patienten mit CVAD auf eine Infektion hinweisen, ohne dass ein anderer Infektionsfokus gefunden werden kann [7]. Die zusätzlichen Symptome grenzen dieses Ereignis vom Fieber unklaren Ursprungs (FUO) ab. Diese infektionsepidemiologisch ausreichende Definition [132] sichert keinesfalls den CVAD als Quelle der klinischen Symptomatik (ggf. auch der positiven Blutkultur). CVAD-assoziierte Infektionen können von gesicherten Infektion des CVAD mit Hilfe der in Tab. 1 aufgeführten Kriterien [48, 92] unterschieden werden.
Zur Diagnose der Lokalinfektion erfolgen Abstriche von der zuvor nicht antiseptisch behandelten Austrittstelle des infizierten Katheters. Wenn die Austrittstelle verkrustet ist, sollte die Kruste vorsichtig mit steriler Kochsalzlösung entfernt werden und der Abstrich vom Wundgrund erfolgen. Die routinemäßige Abnahme von Blutkulturen aus dem Katheter bei symptomlosem Patient oder von Abstrichen einer klinisch unauffälligen Eintrittsstelle ist - außer bei Patienten unter Stammzelltransplantation [29] - eine Vergeudung von Ressourcen [25, 104].

Die Sicherung des Katheters als Quelle der Bakteriämie setzt nach den in Tab. 1 genannten Kriterien entweder die Entfernung des Katheters oder die Abnahme von quantitativen Blutkulturen vor Beginn der Antibiotikatherapie voraus. Der CVAD kann jedoch nicht ohne erheblichen Aufwand (Vollnarkose, meist plus vorübergehende Anlage eines konventionellen ZVK) gewechselt werden. Daher wird nahezu immer erst der Versuch einer „insitu“-Therapie über den liegenden Katheter unternommen [48, $49,52,92,121$ ] Somit scheiden semiquantitative Kulturen der Katheterspitze zum Beweis einer CVAD-Infektion aus [17, 20, 32, 124, 130]. Das Gleiche gilt nach Explantation eines CVAD, da der Katheter praktisch immer zuvor mit antibakterieller Chemotherapie durchspült wurde [64]. Sensitiver als die Katheterspitze kann beim explantierten Port die Kultur von Ablagerungen aus dem Reservoir sein [88].

Um den Ursprung einer Bakteriämie mit größerer Spezifität dem nicht explantierten CVAD zuordnen zu können, wurden verschiedene Methoden der simultanen Kultur zentralvenöser und periphervenöser Blutkulturen vorgeschlagen [17, 20, 22, 27, 48, $118,126]$. In der pädiatrischen Onkologie ergeben sich in diesem Zusammenhang relevante Probleme, die nach Abwägung des zu erwartenden Nutzens gegen die routinemäßige Abnahme zusätzlicher periphervenöser Kulturen sprechen. Die Abnahme

Tab. 1 Verschiedene Formen der CVAD-Infektion [17, 29, 48, 88, $92,110,111,136]$

\begin{tabular}{|c|c|c|}
\hline sichere Infektion & $\begin{array}{l}\text { wahrscheinliche } \\
\text { Infektion }\end{array}$ & mögliche Infektion ${ }^{1}$ \\
\hline $\begin{array}{l}\text { Nachweis des gleichen } \\
\text { Erregers an Katheter- } \\
\text { spitze (in der Port- } \\
\text { kammer- oder tasche) } \\
\text { und in der BK }\end{array}$ & $\begin{array}{l}\text { lokale Infektion an } \\
\text { Austrittstelle und posi- } \\
\text { tive BK }\end{array}$ & $\begin{array}{l}\text { Nachweis eines } \\
\text { "typischen“ Erregers } \\
\text { von CVAD-Infektionen } \\
\text { in BK }{ }^{2}\end{array}$ \\
\hline $\begin{array}{l}\text { DTP (zwischen zentra- } \\
\text { ler und peripher- } \\
\text { venöser Blutkultur) } \\
\text { > } 2 \text { Stunden }\end{array}$ & $\begin{array}{l}\text { positive BK und An- } \\
\text { sprechen von refraktä- } \\
\text { rem Fieber innerhalb } \\
\text { von } 48 \mathrm{~h} \text { nach Kathe- } \\
\text { terentfernung }\end{array}$ & $\begin{array}{l}\text { positive BK und } \\
\text { fehlender anderer } \\
\text { Fokus bei Patient mit } \\
\text { CVAD }\end{array}$ \\
\hline $\begin{array}{l}\text { quantitative Blutkul- } \\
\text { tur: CFU aus Kathe- } \\
\text { ter } \geq \text { Faktor } 10 \text { höher } \\
\text { als CFU in paralleler } \\
\text { periphervenöser BK }\end{array}$ & $\begin{array}{l}\text { Kolonisation der } \\
\text { Katheterspitze in der } \\
\text { quantitativen Kultur } \\
\text { oberhalb der für die } \\
\text { Methode festgelegten } \\
\text { Grenze (meist } \\
\text { >15 CFU) }\end{array}$ & \\
\hline
\end{tabular}

1 Diese Form entspricht der „CVAD-assoziierten Infektion“.

2 Handelt es sich um Hautkeime wie CoNS, so ist der Nachweis in zwei konsekutiven Kulturen (zumindest in zwei Blutkulturflaschen) erforderlich. Abkürzungen: $\mathrm{BK}=$ Blutkultur, CFU = colony forming units, DTP = „differential time to positivity 
der periphervenösen Blutkultur bei gut rückläufigem CVAD ist vielerorts außerhalb wissenschaftlicher Studien nicht üblich. Die quantitative Auswertung der Kulturen ist in der Regel nicht in der Routine verfügbar. Das Risiko einer Kontamination der periphervenösen Kultur mit Hautkeimen beim nicht kooperativen Kind ist erhöht. Zudem ist die Akzeptanz für die zusätzliche Blutentnahme gering, wenn sie auf das therapeutische Vorgehen in den meisten Fällen keinen Einfluss hat [1]. Im St. Jude Children's Hospital (Boston) wurden wahrscheinlich aus den genannten Gründen - trotz einer schriftlich fixierten „hospital-wide policy“ - von 1996-2001 nur in 58\% der Fälle bei fiebernden granulozytopenischen Patienten mit CVAD auch periphervenöse Kulturen abgenommen [53].

Ohne periphere Punktion kann aus der zentral entnommenen Blutprobe die Diagnose durch den direkten Nachweis von Erregern mit dem Acridinorange-Leukozyten-Cytospin-Test gestellt werden [81]. In einer Metaanalyse konnte gezeigt werden, dass dieses Verfahren eine gute Spezifität bei ausreichender Sensitivität aufweist [122]. Es ist jedoch zu aufwändig für die klinische Routine. Möglicherweise werden in Zukunft spezielle Hilfsmittel zur Durchführung von In-situ-Kulturen aus dem Lumen mit einer geschützten Bürste verfügbar sein [42].

Weitere diagnostische Methoden ohne periphere Blutentnahmen wurden von Franklin et al. beschrieben. Sie fanden bei 76 von 136 Bakteriämien (55,9\%) bei Patienten mit doppellumigem Broviac/Hickman in einem Schenkel eine mind. 5-fach höhere Anzahl der CFU/ml und konnten so den infizierten Schenkel mit einem sehr guten positiven Vorhersagewert von 92,2\% identifizieren [53]. Die gleiche Arbeitsgruppe fand für den Schwellenwert von $>100 \mathrm{CFU} / \mathrm{ml}$ aus mind. einem Lumen des CVAD eine Sensitivität, Spezifität und einen positiven prädiktiven Wert von $75,5,69,1$ und $79,3 \%$. Diese Werte sind jedoch zu niedrig für eine breite Anwendung.

Minimalanforderungen zur Diagnosesicherung beinhalten somit die Abnahme von mindestens 2 Blutkulturen (z.B. eine $10 \mathrm{ml}$ Spritze aufteilen auf aerob, anaerob), um die Wahrscheinlichkeit einer Kontamination insbesondere durch CoNS und Corynebakterien zu senken. Bei mehrlumigen CVAD sollte vor Beginn der Therapie aus jedem Lumen eine Blutkultur entnommen werden, da sich die Ergebnisse in bis zu 30\% unterscheiden $[1,120]$.

Ein größeres Blutvolumen in einer Bactec ${ }^{\circledR}$-(Paed)-Flasche erhöht die Sensitivität stärker als die separate Kultivierung mehrerer kleiner Proben $[12,59,70]$. Der Heparin- (nicht der Taurolidin- oder der EDTA ${ }^{3}$ )-Block kann mit in die Kulturflasche gegeben werden $[47,129]$. Die Verwendung von aeroben und anaeroben Blutkultursets kann die Nachweisrate bei fakultativ anaeroben Keimen erhöhen und ist bei immunsupprimierten Patienten zu empfehlen $[12,86]$.

\footnotetext{
${ }^{3}$ Das antimikrobiell wirksame EDTA wird von der Arbeitsgruppe von Isaam Raad (MD Anderson Cancer Center, Houston, Texas) zum Blocken des Katheters verwendet.
}

\section{Wie kann der CVAD als Infektionsquelle gesichert werden?}

Wie oben bereits erläutert kommen die im Folgenden genannten Methoden in der pädiatrischen Routinversorgung nicht zur Anwendung. Ein deutlicher Hinweis für den CVAD als Infektionsquelle ist die vergleichende quantitative Blutkultur [67], wenn der gleiche Erreger im Vergleich mit einer parallel kultivierten periphervenösen Blutprobe mit einer mindestens um den Faktor 5 höheren Koloniezahl in der aus dem CVAD entnommenen Kultur nachgewiesen wird [53, 92]. Eine andere, weniger aufwändige Methode ist die Bestimmung der zentralvenösen vs. periphervenösen Differential Time to Positivity (DTP), d. h. der Zeitspanne zwischen dem Abnahmezeitpunkt und dem ersten positiven Signal im Blutkulturautomaten [17-19]. Ist die DTP der zentralen Blutkultur mindestens 120 min kürzer als die der peripheren, weist dies auf eine Katheterinfektion hin [66, 115]. Blot et al. fanden in einer prospektiven Studie für diese Methode eine Sensitivität von 94\% von eine Spezifität von $91 \%$ [19]. Gaur et al. beschreiben in einer prospektiven Studie mit pädiatrischonkologischen Patienten (nur 33 Episoden) eine Sensitivität von $88,9 \%$ (Spezifität von $100 \%$, positiver prädiktiver Wert $100 \%$, negativer prädiktiver Wert 89-96\%) [58]. Vorstellbar ist der prospektive Einsatz der DTP zur Evaluation eines Infektionsausbruchs, wenn dieser einer erhöhten Inzidenz von CVAD-Infektionen zugeschrieben wird.

In diesem Fall sollte auch eine Typisierung der vermeintlich epidemischen Isolate aus den Blutkulturen (z. B. mittels PFGE) erfolgen $[103,126]$.

\section{Lohnt der Versuch einer In-situ-Therapie?}

Die meisten Episoden einer CVAD-assoziierten Bakteriämie, insbesondere die durch CoNS (bzw. MRSE) verursachten - können ohne Explantation des dauerhaften CVAD geheilt werden.

In zwei prospektiven Studien zur empirischen antibakteriellen Chemotherapie wurde nur bei 10 von insgesamt 500 Episoden (2\%) einer CVAD-assoziierten febrilen Neutropenie die chirurgische Explantation des CVAD im Verlauf erforderlich, um eine Entfieberung des Patienten zu erreichen [50, 51]. Im Verlauf einer prospektiven Surveillancestudie nosokomialer Infektionen (Onkopäd-Pilotstudie) mussten während des 10-monatigen Studienzeitraumes 2 von 32 Broviac-Kathetern (6,3\%) und 4 von 60 Ports $(6,7 \%)$ bei erfolgloser In-situ-Behandlung explantiert werden [132]. Ist der CVAD als Quelle der Bakteriämie gesichert, sind die Heilungsraten niedriger als bei der "nur“ CVAD-asoziierten Bakteriämie. Beim Nachweis von CoNS sind Heilungsraten bis zu $90 \%$ beschrieben $[64,121]$.

\section{Therapie}

Pädiatrisch-onkologische Patienten mit V.a. eine Infektion des CVAD gehören in die kontinuierliche Obhut von erfahrenen pädiatrischen Onkolog(inn)en und sollten umgehend in das zuständige Behandlungszentrum verlegt werden [138]. Oberflächliche Entzündungen an der Katheteraustrittsstelle lassen sich bei nicht granulozytopenischen Patienten durch täglichen 


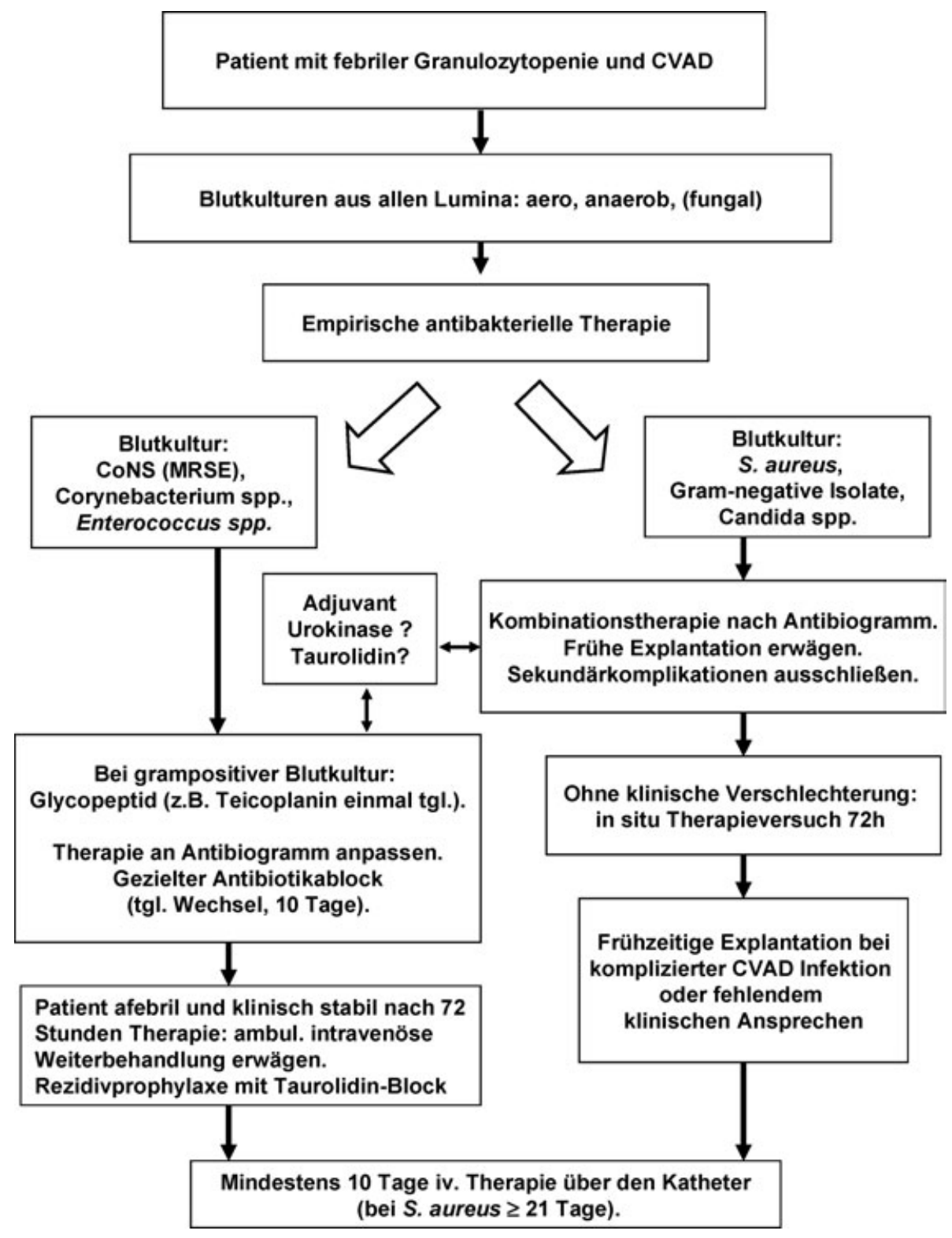

Abb. 1 Orientierender therapeutischer Algorithmus für Patienten mit Fieber in stabilem Allgemeinzustand.

Blutkulturen aus allen Lumina: aero, anaerob, (fungal)

Empirische antibakterielle Therapie 
Die gesicherte Infektion des CVAD (nicht der einfache Nachweis in der Blutkultur) durch bestimmte Erregerspezies, wie z.B. S. aureus [48, 80, 92, 116], Pseudomonas aeruginosa [68], multiresistente Acinetobacter baumannii, atypische Mykobakterien [109] erfordert die umgehende Entfernung des Systems, da die Heilungsraten niedrig sind und das Risiko septischer Komplikationen hoch ist. Die hohen konservativen Heilungsraten (67\%) bei Rubin et al. für CVAD-assoziierte Infektionen durch S. aureus müssen im Licht der Fallzahl von n = 6 mit größter Vorsicht interpretiert werden [121].

Die unkomplizierte CVAD-Infektion spricht innerhalb von 72 Stunden auf die empirische oder gezielte Therapie klinisch und mikrobiologisch (mind. zwei sterile Blutkulturen im Verlauf) an. Einen orientierenden Algorithmus für nicht intensivpflichtige Patienten zeigt Abb. 1. Das in einem abteilungseigenen Standard schriftlich fixierte Behandlungsschema sollte sich Synergieeffekte zwischen Antibiotika mit unterschiedlichem Wirkungsmechanismus zunutze machen (z.B. Teicoplanin und Netilmicin oder Fosfomycin bei grampositiven Erregern oder Ceftazidim und Amikacin bei den gramnegativen). Nach einem Intervall ohne Antibiotikatherapie sollte die Blutkultur erneut kontrolliert werden. Im Sinne einer Sekundärprophylaxe kann versucht werden, das erhöhte Risiko von Reinfektionen [111] durch den Einsatz antimikrobieller Blocklösungen zu senken [15, 75 , 82].

\section{Therapiedauer}

Eine systemische antimikrobielle Therapie sollte bei komplizierten Infektionen und bei Patienten mit hochgradiger Granulozytopenie über 10 Tage, bei Nachweis von S. aureus über mindestens 21 Tage [108, 116], bei infizierter Thrombose und bei Endokarditis (bakterizide Kombinationstherapie) über 4-6 Wochen und bei Osteomyelitis über 6-8 Wochen erfolgen [61, 89]. Vor einer erneuten Implantation eines dauerhaften CVAD sollten mindestens zwei negative Blutkulturen die Eradikation des Erregers belegt haben $[48,49]$. Eine Beteiligung des Endokards und eine Thrombose der zentralen Venen sind auszuschließen. Bei CVADassoziierten Pilzinfektionen ${ }^{4}$ sollte die Therapie mindestens 14 Tage nach Entnahme der ersten negativen Blutkultur und Besserung der klinischen Symptomatik andauern [105].

\section{Adjuvante Therapiemaßnahmen}

Die im Folgenden genannten adjuvanten Therapietechniken (Antibiotikablock, Ethanol, Taurolidin, Urokinase) sind aufgrund von pathogenetischen Überlegungen, Fallberichten und offenen kontrollierten Studien vielerorts bereits gängige Praxis, ohne dass prospektiv-kontrollierte, doppelblinde Studien zu ihrem Nutzen vorliegen. Je nach Erfahrung und Einschätzung durch die verantwortlichen Onkologen kann ihr Einsatz von erheblichem Nutzen sein, wobei auch hier ein standardisiertes Vorgehen anzustreben ist.

\footnotetext{
${ }^{4}$ Siehe Übersicht von A. Groll in diesem Band
}

\section{Antibiotikablocktechnik (ALT)}

Die Richtlinien der Infectious Diseases Society of America [92] empfehlen bei unkomplizierten Katheter-assoziierten Bakteriämien durch CoNS, S. aureus und gramnegative Erreger ${ }^{5}$ die Blockung des Katheters für 14 Tage (täglicher Wechsel) in Ergänzung zu einer 7-tägigen systemisch verabreichten antibakteriellen Therapie. Die kürzlich von Bestul und Vandenbusche publizierte Metaanalyse sieht den Nutzen einer routinemäßigen ALT nicht als erwiesen an [14].

Der Vorteil der Instillation in das Broviaclumen (in das Portreservoir) besteht in einer deutlich höheren Konzentration und Wirkdauer des Antibiotikums am Ort der Infektion (oder Besiedlung) ohne systemische Nebenwirkungen $[9,13,14,23,24,33,39,45$, $64,69,146]$. Besonders viel versprechend ist dieses von Messing et al. für Patienten mit heimparenteraler Ernährung entwickelte Konzept [93] in der Supprimierung („unentbehrlicher Zugang“) oder Eradikation von opportunistischen, Biofilmbildenden Bakterien wie CoNS $[33,39,119,146]$. Allerdings darf nicht übersehen werden, dass trotz einer solchen Intervention echte Rezidive (erneute Infektion mit einem genotypisch identischen Isolat) keine Seltenheit sind, so dass es sich hier mitunter nicht um eine Eradikation handelt [62].

Natürlich dürfen extraluminale (lokale oder systemische) CVADassoziierte Infektionen nicht mit einer ALT behandelt werden, sondern sollten zumindest in den ersten 72 Stunden immer mit einer systemischen Behandlung kombiniert werden. Die kurzfristigen Erfolgsraten bei Erwachsenen mit unterschiedlichen Grunderkrankungen und alleiniger ALT oder kombiniert mit systemischer Therapie reichen von 30-100\% [9, 13, 14, 142].

Hinsichtlich der optimalen Konzentration und intraluminalen Verweildauer der ALT kann zur Zeit noch keine evidenzbasierte Empfehlung ausgesprochen werden [92]. Linezolid (2 mg/ml) scheint in Biofilm eingeschlossene CoNS schneller abzutöten als Vancomycin $(10 \mathrm{mg} / \mathrm{ml})$ oder Gentamicin $(10 \mathrm{mg} / \mathrm{ml})$ [34, 45, 146]. In vitro sind gegen biofilmbildene CoNS Kombinationen von Antibiotika, insbesondere Minocyclin plus Vancomycin, Minocyclin plus Rifampicin, Vancomycin plus Rifampicin sowie Minocyclin plus EDTA [28, 91, 112 - 114] deutlich wirksamer sind als Einzelsubstanzen. Rifampicin und Minocyclin haben den (auf die mögliche Selektion resistenter Isolate bezogenen) Vorteil, dass sie in der gezielten Behandlung bakterieller Infektionen in dieser Patientengruppe praktisch keine Rolle spielen.

Die Stabilität von verschiedenen Antibiotika-Heparin-Mischungen (siehe Tab. 2) konnte in vitro und in vivo dokumentiert werden $[5,35,45,65,146]$. Die Kombination von Vancomycinlösung $(25 \mu \mathrm{g} / \mathrm{ml} \mathrm{d.h.} 2,5 \mathrm{mg}$ auf $100 \mathrm{ml} \mathrm{NaCl} 0,9 \%)$ und Heparin $100 \mathrm{E} / \mathrm{ml}$ (10 $000 \mathrm{E}$ auf $100 \mathrm{ml} \mathrm{NaCl}$ 0,9\%) ist nach eigenen Untersuchungen über mindestens 14 Tage stabil und weiterhin bakterizid. Über 7 Tage stabil (mit $100 \mathrm{E}$ Heparin/ml) sind z. B. Vancomycin, Cefazolin, Ceftazidim (Konzentration jeweils $500 \mu \mathrm{g}=$ $0,5 \mathrm{mg} / \mathrm{ml}$ ) und Ciprofloxacin (Konz. $125 \mu \mathrm{g} / \mathrm{ml}$ ) [siehe Tab. 2 und Übersicht bei Trautmann [142]].

\footnotetext{
${ }^{5}$ Nicht übereinstimmend mit den Empfehlungen zu Infektionen durch S. aureus oder P. aeruginosa in diesem Artikel, siehe Text.
} 
Tab. 2 Antimikrobielle Blocklösungen [5, 35, 45, 65, 142, 146]

\begin{tabular}{|c|c|}
\hline Wirkstoff & Konzentration (mg/ml) \\
\hline Vancomycin ${ }^{1}$ & $0,025-10$ \\
\hline Teicoplanin ${ }^{1}$ & $0,025-2,5-20$ \\
\hline Linezolid ${ }^{1}$ & $0,2-2$ \\
\hline Amikacin ${ }^{1,2}$ & $1-10$ \\
\hline Gentamicin & $1-10$ \\
\hline Ciprofloxacin & $0,125-2$ \\
\hline Ceftazidim & $0,5-2$ \\
\hline Amphotericin B Desoxycholat & 2 (in Glukose 5\%) \\
\hline \multicolumn{2}{|c|}{$\begin{array}{l}{ }^{1} \text { stabil über mind. } 24 \text { Stunden ohne Wirkverlust in Kombination mit Heparin } \\
100 \mathrm{E} / \mathrm{ml} \\
{ }^{2} \text { Vancomyin } 25 \mu \mathrm{g} / \mathrm{ml}+\text { Amikacin } 25 \mu \mathrm{g} / \mathrm{ml}+\text { Heparin } 100 \mathrm{E} / \mathrm{ml} \text { in } \mathrm{NaCl} 0,9 \% \\
\text { [35] } \\
\text { Hinweis: Von der Klinikapotheke bereitgestellte Standardampullen zur ALT } \\
\text { müssen sorgfältig vor Kontamination durch Bakterien und Pilze geschützt, bei } \\
\text { Vorratshaltung steril filtriert und im Kühlschrank gelagert werden. }\end{array}$} \\
\hline
\end{tabular}

\section{Ethanolinstillation}

Dannenberg u. Mitarb. (Arbeitsgruppe Prof. Körholz, Universitätskinderklinik Leipzig) berichten über positive Erfahrungen mit Ethanol-Instillationen (2,3 ml einer 74\%igen Ethanollösung, Verweildauer 20-24Stunden) bei luminal infizierten BroviacKathetern [37]. Lediglich bei einer von 39 Episoden (28 Patienten) musste der Broviac im Verlauf der Infektion explantiert werden. Der Alkoholblock darf nicht aspiriert werden, da sich der Katheter sonst sofort durch denaturiertes Blut verschließt. Nach dem Ethanolflush kam es bei zu leichten Symptomen, wie Müdigkeit, vorübergehender Schwindel, Kopfschmerzen.

\section{Taurolidin}

Taurolidin ist ein Derivat der biogenen Aminosäure Taurin. Taurolidin zeigt in vitro ein breites bakterizides und fungizides (Candida spp.) Wirkungsspektrum [127, 140, 141]. Im Unterschied zur Anwendung von antibiotikahaltigen Blocklösungen ist die Anwendung von Taurolidin nicht abhängig vom Resistenzprofil der Isolate gegenüber Antibiotika (auch MRSE, MRSA, VRE, ESBL-bildende Isolate). Die Selektion antibiotikaresistenter Mikroorganismen durch den Einsatz von Taurolidin ist nicht zu befürchten. In der Primärprophylaxe scheint Taurolidin die Ausbildung des intraluminalen Biofilms zu verhindern [4, 127]. Ob Taurolidin einen bereits ausgebildeten Biofilm durchdringt, ist nicht ausreichend untersucht [36].

Da Taurolidin zu Taurinamid, Taurin und $\mathrm{CO}_{2}$ metabolisiert wird, ist es bei i.v. Anwendung nicht toxisch. Johnston et al. setzen Taurolidin der parenteralen Ernährung zu (10g auf 31, 0,3\%) und unterbrachen so eine ständige Abfolge von schweren Katheterinfektionen mit Endokarditis bei einer 26-jährigen Patienten (Kurzdarm bei M. Crohn) [71]. Ein Taurolidin-Block ${ }^{6}$ kann

\footnotetext{
${ }^{6}$ TauroLock $^{\mathrm{TM}}$, 5-ml-Ampullen, 1,35\% Taurolidin, 4\% Citrat, TauroPharm $\mathrm{GmbH}$, Waldbüttelbrunn
}

zur Sekundärprophylaxe [75] und begleitend zur Antibiotikagabe auch in der Therapie [82] von CVAD-Infektionen eingesetzt werden.

\section{Urokinase}

Nach eigener, nicht kontrollierter Erfahrung (AS) erhöht die Instillation von Urokinase in den CVAD vor Abnahme die Sensitivität der Blutkultur. Die für den Einsatz von Urokinase erforderliche ärztliche Anordnung wird durch die Einführung eines abteilungseigenen Urokinase-Standards vereinfacht. Urokinase (Urokinase HS medac ${ }^{\circledR} 10000$ I.E.) wird mit sterilem Aqua dest. verdünnt ( $4 \mathrm{ml}=2500$ I.E. $/ \mathrm{ml} ; 2 \mathrm{ml}=5000$ I.E. $/ \mathrm{ml} ; 1 \mathrm{ml}=10000$ I.E./ $\mathrm{ml}$ ). Das instillierte Volumen sollte nicht größer sein, als das Lumen des CVAD. Bei doppellumigen Systemen ist die Gesamtdosis auf beide Lumina zu verteilen. Ergebnisse von in-vitro-Experimenten, Extrapolationen aus Prophylaxestudien [41, 76] sowie Anwendungsbeobachtungen und klinische Studien [39] sprechen für den adjuvanten Einsatz von Urokinase bei grampositiven Katheterinfektionen (Übersicht bei Kellermann [77]). Das in der Abteilung für Pädiatrische Hämatologie und Onkologie des Universitätsklinikums Bonn übliche Dosierungsschema lautet: $3-10 \mathrm{~kg} 2500 \mathrm{E}$, über $10 \mathrm{~kg} 5000 \mathrm{E}$ und über $25 \mathrm{~kg} 10000 \mathrm{E}$. Nach Ablauf der geplanten Verweildauer von mindestens 4 Stunden wird unter sterilen Bedingungen der Urokinaseblock aspiriert und für eine aerobe Blutkultur verwendet. Dann wird der ZVK mit $\mathrm{NaCl}$ 0,9\%/Heparin $10 \mathrm{E} / \mathrm{ml}$ gespült und sofort an die Antibiotikainfusion angeschlossen. Eine Wiederholung dieser Prozedur nach 24 Stunden ist sinnvoll. Jones et al. konnten auf diese Weise 59 von 67 (88\%) CVAD-assoziierten, grampositiven Bakteriämien erfolgreich in situ behandeln [72, 73].

\section{Literatur}

${ }^{1}$ Adamkiewicz TV, Lorenzana A, Doyle J et al. Peripheral vs. central blood cultures in patients admitted to a pediatric oncology ward. Pediatr Infect Dis J 1999; 18: 556 - 558

2 Aledo A, Heller G, Ren L et al. Septicemia and septic shock in pediatric patients: 140 consecutive cases on a pediatric hematology-oncology service. J Pediatr Hematol Oncol 1998; 20: 215 - 221

${ }^{3}$ Allon M. Dialysis catheter-related bacteremia: treatment and prophylaxis. Am J Kidney Dis 2004; 44: 779-791

${ }^{4}$ Allon M. Prophylaxis against dialysis catheter-related bacteremia with a novel antimicrobial lock solution. Clin Infect Dis 2003; 36: $1539-1544$

${ }^{5}$ Anthony TU, Rubin LG. Stability of antibiotics used for antibiotic-lock treatment of infections of implantable venous devices (ports). Antimicrob Agents Chemother 1999; 43: 2074-2076

${ }^{6}$ Ascher DP, Shoupe BA, Maybee D et al. Persistent catheter-related bacteremia: clearance with antibiotics and urokinase. J Pediatr Surg 1993; 28: 627-629

${ }^{7}$ Auletta JJ, O'Riordan MA, Nieder ML. Infections in children with cancer: a continued need for the comprehensive physical examination. J Pediatr Hematol Oncol 1999; 21: $501-508$

8 Babu R, Spicer RD. Implanted vascular access devices (ports) in children: complications and their prevention. Pediatr Surg Int 2002; 18: $50-53$

${ }^{9}$ Bagnall-Reeb H. Evidence for the use of the antibiotic lock technique. J Infus Nurs 2004; 27: 118 -122

10 Beghetti M, La Scala G, Belli D et al. Etiology and management of pediatric chylothorax. J Pediatr 2000; 136: 653-658

${ }^{11}$ Berner R, Sauter S, Michalski Y et al. Central venous catheter infection by Aspergillus fumigatus in a patient with B-type non-Hodgkin lymphoma. Med Pediatr Oncol 1996; 27: $202-204$ 
12 Berner R, Schumacher RF, Bartelt S et al. Predisposing conditions and pathogens in bacteremia in hospitalized children. Eur J Clin Microbiol Infect Dis 1998; 17: $337-340$

${ }^{13}$ Berrington A, Gould FK. Use of antibiotic locks to treat colonized central venous catheters. J Antimicrob Chemother 2001; 48: 597-603

${ }^{14}$ Bestul MB, Vandenbussche HL. Antibiotic lock technique: review of the literature. Pharmacotherapy 2005; 25: 211 -227

${ }^{15}$ Betjes MG, van Agteren M. Prevention of dialysis catheter-related sepsis with a citrate-taurolidine-containing lock solution. Nephrol Dial Transplant 2004; 19: 1546 - 1551

${ }^{16}$ Biagi E, Arrigo C, Dell'Orto MG et al. Mechanical and infective central venous catheter-related complications: a prospective non-randomized study using Hickman and Groshong catheters in children with hematological malignancies. Support Care Cancer 1997; 5: 228-233

17 Blot F, Nitenberg G, Brun-Buisson C. New tools in diagnosing catheter-related infections. Support Care Cancer 2000; 8: 287-292

18 Blot F, Nitenberg G, Chachaty E et al. Diagnosis of catheter-related bacteraemia: a prospective comparison of the time to positivity of hub-blood versus peripheral-blood cultures. Lancet 1999; 354: 1071 1077

${ }^{19}$ Blot F, Schmidt E, Nitenberg G et al. Earlier positivity of central-venous- versus peripheral-blood cultures is highly predictive of catheter-related sepsis. J Clin Microbiol 1998; 36: 105-109

${ }^{20}$ Bouza E, Burillo A, Munoz P. Catheter-related infections: diagnosis and intravascular treatment. Clin Microbiol Infect 2002; 8: 265-274

${ }^{21}$ Cairo MS, Spooner S, Sowden L et al. Long-term use of indwelling multipurpose silastic catheters in pediatric cancer patients treated with aggressive chemotherapy. J Clin Oncol 1986; 4: 784- 788

${ }^{22}$ Capdevila JA, Planes AM, Palomar M et al. Value of differential quantitative blood cultures in the diagnosis of catheter-related sepsis. Eur J Clin Microbiol Infect Dis 1992; 11: $403-407$

${ }^{23}$ Carratala J. Role of antibiotic prophylaxis for the prevention of intravascular catheter-related infection. Clin Microbiol Infect 2001; 7 (Suppl 4): 83-90

24 Carratala J, Niubo J, Fernandez-Sevilla A et al. Randomized, doubleblind trial of an antibiotic-lock technique for prevention of grampositive central venous catheter-related infection in neutropenic patients with cancer. Antimicrob Agents Chemother 1999; 43: $2200-$ 2204

25 Catton JA, Dobbins BM, Wood JM et al. The routine microbiological screening of central venous catheters in home parenteral nutrition patients. Clin Nutr 2004; 23: $171-175$

${ }^{26}$ Cesaro S, Corro R, Pelosin A et al. A prospective survey on incidence and outcome of Broviac/Hickman catheter-related complications in pediatric patients affected by hematological and oncological diseases. Ann Hematol 2004; 83: $183-188$

27 Chatzinikolaou I, Hanna H, Hachem R et al. Differential quantitative blood cultures for the diagnosis of catheter-related bloodstream infections associated with short- and long-term catheters: a prospective study. Diagn Microbiol Infect Dis 2004; 50: 167-172

${ }^{28}$ Chatzinikolaou I, Zipf TF, Hanna $\mathrm{H}$ et al. Minocycline-ethylenediaminetetraacetate lock solution for the prevention of implantable port infections in children with cancer. Clin Infect Dis 2003; 36: $116-119$

${ }^{29}$ Chizuka A, Kami M, Kanda Y et al. Value of surveillance blood culture for early diagnosis of occult bacteremia in patients on corticosteroid therapy following allogeneic hematopoietic stem cell transplantation. Bone Marrow Transplant 2005; 35: 577-582

${ }^{30}$ Christensen ML, Hancock ML, Gattuso J et al. Parenteral nutrition associated with increased infection rate in children with cancer. Cancer 1993; 72: $2732-2738$

${ }^{31}$ Cisneros JM, Rodriguez-Bano J. Nosocomial bacteremia due to Acinetobacter baumannii: epidemiology, clinical features and treatment. Clin Microbiol Infect 2002; 8: 687-693

32 Cohen J, Brun-Buisson C, Torres A et al. Diagnosis of infection in sepsis: an evidence-based review. Crit Care Med 2004; 32: S466-S 494

33 Cuntz D, Michaud L, Guimber D et al. Local antibiotic lock for the treatment of infections related to central catheters in parenteral nutrition in children. JPEN J Parenter Enteral Nutr 2002; 26: 104-108

${ }^{34}$ Curtin J, Cormican M, Fleming G et al. Linezolid compared with eperezolid, vancomycin, and gentamicin in an in vitro model of antimicrobial lock therapy for Staphylococcus epidermidis central venous catheter-related biofilm infections. Antimicrob Agents Chemother 2003; 47: $3145-3148$
${ }^{35}$ Daghistani D, Horn M, Rodriguez Z et al. Prevention of indwelling central venous catheter sepsis. Med Pediatr Oncol 1996; 26: $405-$ 408

${ }^{36}$ Danese PN. Antibiofilm approaches: prevention of catheter colonization. Chem Biol 2002; 9: 873-880

37 Dannenberg C, Bierbach U, Rothe A et al. Ethanol-lock technique in the treatment of bloodstream infections in pediatric oncology patients with broviac catheter. J Pediatr Hematol Oncol 2003; 25: $616-621$

38 Dato VM, Dajani AS. Candidemia in children with central venous catheters: role of catheter removal and amphotericin B therapy. Pediatr Infect Dis J 1990; 9: 309-314

39 De Sio L, Jenkner A, Milano GM et al. Antibiotic lock with vancomycin and urokinase can successfully treat colonized central venous catheters in pediatric cancer patients. Pediatr Infect Dis J 2004; 23: 963 - 965

${ }^{40}$ Dettenkofer M, Jonas D, Wiechmann C et al. Effect of skin disinfection with octenidine dihydrochloride on insertion site colonization of intravascular catheters. Infection 2002; 30: $282-285$

${ }^{41}$ Dillon PW, Jones GR, Bagnall-Reeb HA et al. Prophylactic urokinase in the management of long-term venous access devices in children: a Children's Oncology Group study. J Clin Oncol 2004; 22: 2718-2723

42 Dobbins BM, Kite P, Catton JA et al. In situ endoluminal brushing: a safe technique for the diagnosis of catheter-related bloodstream infection. J Hosp Infect 2004; 58: $233-237$

${ }^{43}$ Donnell SC, Taylor N, Saene HK van. Translocation cannot be ignored during parenteral nutrition. J Hosp Infect 2004; 56: 246 - 247

${ }^{44}$ Donnell SC, Taylor N, Saene HK van et al. Infection rates in surgical neonates and infants receiving parenteral nutrition: a five-year prospective study. J Hosp Infect 2002; 52: $273-280$

45 Droste JC, Jeraj HA, MacDonald A et al. Stability and in vitro efficacy of antibiotic-heparin lock solutions potentially useful for treatment of central venous catheter-related sepsis. J Antimicrob Chemother 2003; 51: $849-855$

${ }^{46}$ Eppes SC, Troutman JL, Gutman LT. Outcome of treatment of candidemia in children whose central catheters were removed or retained. Pediatr Infect Dis J 1989; 8: 99- 104

47 Everts R, Harding H. Catheter-drawn blood cultures: is withdrawing the heparin lock beneficial? Pathology 2004; 36: 170-173

48 Fatkenheuer G, Buchheidt D, Cornely OA et al. Central venous catheter (CVC)-related infections in neutropenic patients - guidelines of the Infectious Diseases Working Party (AGIHO) of the German Society of Hematology and Oncology (DGHO). Ann Hematol 2003; 82 (Suppl 2): S 149-S157

${ }^{49}$ Fatkenheuer G, Cornely O, Seifert H. Clinical management of catheter-related infections. Clin Microbiol Infect 2002; 8: 545-550

${ }^{50}$ Fleischhack G, Hartmann C, Simon A et al. Meropenem versus ceftazidime as empirical monotherapy in febrile neutropenia of paediatric patients with cancer. J Antimicrob Chemother 2001; 47: 841 -853

${ }^{51}$ Fleischhack G, Schmidt-Niemann M, Wulff B et al. Piperacillin, betalactam inhibitor plus gentamicin as empirical therapy of a sequential regimen in febrile neutropenia of pediatric cancer patients. Support Care Cancer 2001; 9: 372 - 379

52 Flynn PM, Shenep JL, Stokes DC et al. In situ management of confirmed central venous catheter-related bacteremia. Pediatr Infect Dis J 1987; 6: 729-734

${ }^{53}$ Franklin JA, Gaur AH, Shenep JL et al. In situ diagnosis of central venous catheter-related bloodstream infection without peripheral blood culture. Pediatr Infect Dis J 2004; 23: 614-618

${ }^{54}$ Fratino G, Molinari AC, Parodi S et al. Central venous catheter-related complications in children with oncological/hematological diseases: an observational study of 418 devices. Ann Oncol 2005; 16: 648 - 654

${ }^{55}$ Friedman ND, Korman TM, Fairley CK et al. Bacteraemia due to Stenotrophomonas maltophilia: an analysis of 45 episodes. J Infect 2002; 45: $47-53$

${ }^{56}$ Fukunaga A, Naritaka H, Fukaya R et al. Povidone-iodine ointment and gauze dressings associated with reduced catheter-related infection in seriously ill neurosurgical patients. Infect Control Hosp Epidemiol 2004; 25: 696-698

${ }^{57}$ Gassas A, Grant R, Richardson S et al. Predictors of viridans streptococcal shock syndrome in bacteremic children with cancer and stemcell transplant recipients. J Clin Oncol 2004; 22: 1222-1227

58 Gaur AH, Flynn PM, Giannini MA et al. Difference in time to detection: a simple method to differentiate catheter-related from noncatheter-related bloodstream infection in immunocompromised pediatric patients. Clin Infect Dis 2003; 37: 469-475 
${ }^{59}$ Gaur AH, Giannini MA, Flynn PM et al. Optimizing blood culture practices in pediatric immunocompromised patients: evaluation of media types and blood culture volume. Pediatr Infect Dis J 2003; 22: 545-552

${ }^{60}$ Gorelick MH, Owen WC, Seibel NL et al. Lack of association between neutropenia and the incidence of bacteremia associated with indwelling central venous catheters in febrile pediatric cancer patients. Pediatr Infect Dis J 1991; 10: 506-510

${ }^{61}$ Graninger W, Assadian O, Lagler $\mathrm{H}$ et al. The role of glycopeptides in the treatment of intravascular catheter-related infections. Clin $\mathrm{Mi}-$ crobiol Infect 2002; 8: 310-315

${ }^{62}$ Guedon C, Nouvellon M, Lalaude $\mathrm{O}$ et al. Efficacy of antibiotic-lock technique with teicoplanin in staphylococcus epidermidis catheterrelated sepsis during long-term parenteral nutrition. JPEN J Parenter Enteral Nutr 2002; 26: 109-113

${ }^{63}$ Guggenbichler JP, Berchtold D, Allerberger F et al. In vitro and in vivo effect of antibiotics on catheters colonized by staphylococci. Eur J Clin Microbiol Infect Dis 1992; 11: 408-415

${ }^{64}$ Hachem R, Raad I. Prevention and management of long-term catheter related infections in cancer patients. Cancer Invest 2002; 20: 1105 1113

${ }^{65}$ Haimi-Cohen Y, Husain N, Meenan J et al. Vancomycin and ceftazidime bioactivities persist for at least 2 weeks in the lumen in ports: simplifying treatment of port-associated bloodstream infections by using the antibiotic lock technique. Antimicrob Agents Chemother 2001; 45: $1565-1567$

${ }^{66}$ Haimi-Cohen Y, Shafinoori S, Tucci V et al. Use of incubation time to detection in BACTEC 9240 to distinguish coagulase-negative staphylococcal contamination from infection in pediatric blood cultures. Pediatr Infect Dis J 2003; 22: 968 - 974

${ }^{67}$ Haimi-Cohen Y, Vellozzi EM, Rubin LG. Initial concentration of Staphylococcus epidermidis in simulated pediatric blood cultures correlates with time to positive results with the automated, continuously monitored BACTEC blood culture system. J Clin Microbiol 2002; 40: $898-901$

${ }^{68}$ Hanna H, Afif C, Alakech B et al. Central venous catheter-related bacteremia due to gram-negative bacilli: significance of catheter removal in preventing relapse. Infect Control Hosp Epidemiol 2004; 25: $646-649$

${ }^{69}$ Henrickson KJ, Axtell RA, Hoover SM et al. Prevention of central venous catheter-related infections and thrombotic events in immunocompromised children by the use of vancomycin/ciprofloxacin/heparin flush solution: A randomized, multicenter, double-blind trial. J Clin Oncol 2000; 18: 1269-1278

70 Isaacman DJ, Karasic RB, Reynolds EA et al. Effect of number of blood cultures and volume of blood on detection of bacteremia in children. J Pediatr 1996; 128: 190-195

71 Johnston D, Phillips G, Perry M et al. Taurolin for the prevention of parenetral nutrition related infection: antimicrobial activity and long-term use. Clinical Nutrition 1993; 12: 365 - 368

72 Jones GR, Konsler GK, Dunaway RP. Urokinase in the treatment of bacteremia and candidemia in patients with right atrial catheters. Am J Infect Control 1996; 24: 160-166

${ }^{73}$ Jones GR, Konsler GK, Dunaway RP et al. Prospective analysis of urokinase in the treatment of catheter sepsis in pediatric hematologyoncology patients. J Pediatr Surg 1993; 28: 350-355

74 Journeycake JM, Buchanan GR. Thrombotic complications of central venous catheters in children. Curr Opin Hematol 2003; 10: 369-374

75 Jurewitsch B, Lee T, Park J et al. Taurolidine $2 \%$ as an antimicrobial lock solution for prevention of recurrent catheter-related bloodstream infections. JPEN J Parenter Enteral Nutr 1998; 22: 242-244

${ }^{76}$ Kalmanti M, Germanakis J, Stiakaki E et al. Prophylaxis with urokinase in pediatric oncology patients with central venous catheters. Pediatr Hematol Oncol 2002; 19: 173-179

${ }^{77}$ Kellerman S, Chan J, Jarvis W. Use of urokinase in pediatric hematology/oncology patients. Am J Infect Control 1998; 26: 502-506

${ }^{78}$ Kennedy HF, Morrison D, Tomlinson D et al. Gingivitis and toothbrushes: potential roles in viridans streptococcal bacteraemia. J Infect 2003; 46: 67-70

${ }^{79} \mathrm{Kim}$ DH, Bae NY, Sung WJ et al. Hickman catheter site infections after allogeneic stem cell transplantation: a single-center experience. Transplant Proc 2004; 36: 1569-1573

${ }^{80} \mathrm{Kim}$ SH, Kang CI, Kim HB et al. Outcomes of Hickman catheter salvage in febrile neutropenic cancer patients with Staphylococcus aureus bacteremia. Infect Control Hosp Epidemiol 2003; 24: 897-904
${ }^{81}$ Kite P, Dobbins BM, Wilcox MH et al. Rapid diagnosis of central-venous-catheter-related bloodstream infection without catheter removal. Lancet 1999; 354: 1504-1507

82 Koldehoff M, Zakrzewski JL. Taurolidine is effective in the treatment of central venous catheter-related bloodstream infections in cancer patients. Int J Antimicrob Agents 2004; 24: 491 - 495

83 Kramer A, Daeschlein G, Kammerlander G et al. Consensus recommendation for the choice of antiseptic agents in wound care (Article in German). Hygiene und Medizin 2004; 29: 147 - 157

${ }^{84}$ La Quaglia MP, Lucas A, Thaler HT et al. A prospective analysis of vascular access device-related infections in children. J Pediatr Surg 1992; 27: $840-842$

${ }^{85}$ Laws H, Göbel U, Christaras A et al. Intensivierte Chelattherapie bei Patienten mit Thalassemia major. Klin Pädiatr 2005; 217: 120-125

${ }^{86}$ Lee CS, Hwang B, Chung RL et al. The assessment of anaerobic blood culture in children. J Microbiol Immunol Infect 2000; 33: 49-52

${ }^{87}$ Levy I, Rubin LG, Vasishtha S et al. Emergence of Candida parapsilosis as the predominant species causing candidemia in children. Clin Infect Dis 1998; 26: $1086-1088$

${ }^{88}$ Longuet P, Douard MC, Arlet G et al. Venous access port-related bacteremia in patients with acquired immunodeficiency syndrome or cancer: the reservoir as a diagnostic and therapeutic tool. Clin Infect Dis $2001 ; 32: 1776-1783$

${ }^{89}$ McCarthy A, Rao JS, Byrne M et al. Central venous catheter infections treated with teicoplanin. Eur J Haematol Suppl 1998; 62: 15 - 17

${ }^{90}$ McGee DC, Gould MK. Preventing complications of central venous catheterization. N Engl J Med 2003; 348: 1123 - 1133

${ }^{91}$ Mermel LA. Prevention of intravascular catheter-related infections. Ann Intern Med 2000; 132: 391 - 402

92 Mermel LA, Farr BM, Sherertz RJ et al. Guidelines for the management of intravascular catheter-related infections. J Intraven Nurs 2001; 24: $180-205$

${ }_{93}$ Messing B, Peitra-Cohen S, Debure A et al. Antibiotic-lock technique: a new approach to optimal therapy for catheter-related sepsis in home-parenteral nutrition patients. JPEN J Parenter Enteral Nutr 1988; 12 : $185-189$

${ }^{94}$ Mirro Jr J, Rao BN, Kumar M et al. A comparison of placement techniques and complications of externalized catheters and implantable port use in children with cancer. J Pediatr Surg 1990; 25: 120-124

${ }^{95}$ Mirro Jr J, Rao BN, Stokes DC et al. A prospective study of Hickman/ Broviac catheters and implantable ports in pediatric oncology patients. J Clin Oncol 1989; 7: 214-222

${ }^{96}$ Molinari AC, Castagnola E, Mazzola C et al. Thromboembolic complications related to indwelling central venous catheters in children with oncological/haematological diseases: a retrospective study of 362 catheters. Support Care Cancer 2001; 9: 539-544

${ }^{97}$ Molinari AC, Haupt R, Saracco P et al. Urokinase for restoring patency of malfunctioning or blocked central venous catheters in children with hemato-oncological diseases. Support Care Cancer 2004; 12 : $840-843$

${ }^{98}$ Monzon M, Oteiza C, Leiva J et al. Synergy of different antibiotic combinations in biofilms of Staphylococcus epidermidis. J Antimicrob Chemother 2001; 48: 793-801

${ }^{99}$ Monzon M, Oteiza C, Leiva J et al. Biofilm testing of Staphylococcus epidermidis clinical isolates: low performance of vancomycin in relation to other antibiotics. Diagn Microbiol Infect Dis 2002; 44: 319 324

${ }^{100}$ Morrison VA, Peterson BA, Bloomfield CD. Nosocomial septicemia in the cancer patient: the influence of central venous access devices, neutropenia, and type of malignancy. Med Pediatr Oncol 1990; 18: 209-216

101 Mullen CA, Abd El-Baki H, Samir H et al. Non-albicans Candida is the most common cause of candidemia in pediatric cancer patients. Support Care Cancer 2003; 11: 321 - 325

102 Nace CS, Ingle RJ. Central venous catheter "pinch-off" and fracture: a review of two under-recognized complications. Oncol Nurs Forum 1993; 20: 1227 - 1236

103 Nouwen JL, Belkum A van, de Marie S et al. Clonal expansion of Staphylococcus epidermidis strains causing Hickman catheter-related infections in a hemato-oncologic department. J Clin Microbiol 1998; 36: $2696-2702$

104 O'Grady NP, Alexander M, Dellinger EP et al. Guidelines for the prevention of intravascular catheter-related infections. Infect Control Hosp Epidemiol 2002; 23: 759-769 
105 Pappas PG, Rex JH, Sobel JD et al. Guidelines for treatment of candidiasis. Clin Infect Dis 2004; 38: 161 - 189

106 Posteraro B, Bruno S, Boccia S et al. Candida parapsilosis bloodstream infection in pediatric oncology patients: results of an epidemiologic investigation. Infect Control Hosp Epidemiol 2004; 25: 641 - 645

107 Raad II, Luna M, Khalil SA et al. The relationship between the thrombotic and infectious complications of central venous catheters. Jama 1994; 271: 1014 - 1016

108 Raad II, Sabbagh MF. Optimal duration of therapy for catheter-related Staphylococcus aureus bacteremia: a study of 55 cases and review. Clin Infect Dis 1992; 14: 75 - 82

109 Raad II, Vartivarian S, Khan A et al. Catheter-related infections caused by the Mycobacterium fortuitum complex: 15 cases and review. Rev Infect Dis 1991; 13: 1120-1125

110 Raad I. Intravascular-catheter-related infections. Lancet 1998; 351 : $893-898$

111 Raad I. Management of intravascular catheter-related infections. J Antimicrob Chemother 2000; 45: 267-270

112 Raad I, Buzaid A, Rhyne J et al. Minocycline and ethylenediaminetetraacetate for the prevention of recurrent vascular catheter infections. Clin Infect Dis 1997; 25: 149-151

113 Raad I, Chatzinikolaou I, Chaiban G et al. In vitro and ex vivo activities of minocycline and EDTA against microorganisms embedded in biofilm on catheter surfaces. Antimicrob Agents Chemother 2003; 47: $3580-3585$

114 Raad I, Hachem R, Tcholakian RK et al. Efficacy of minocycline and EDTA lock solution in preventing catheter-related bacteremia, septic phlebitis, and endocarditis in rabbits. Antimicrob Agents Chemother 2002; 46: $327-332$

115 Raad I, Hanna HA, Alakech B et al. Differential time to positivity: a useful method for diagnosing catheter-related bloodstream infections. Ann Intern Med 2004; 140: 18-25

116 Raad I, Narro J, Khan A et al. Serious complications of vascular catheter-related Staphylococcus aureus bacteremia in cancer patients. Eur J Clin Microbiol Infect Dis 1992; 11: 675-682

117 Rackoff WR, Ge J, Sather HN et al. Central venous catheter use and the risk of infection in children with acute lymphoblastic leukemia: a report from the Children's Cancer Group. J Pediatr Hematol Oncol 1999; 21: $260-267$

118 Raucher HS, Hyatt AC, Barzilai A et al. Quantitative blood cultures in the evaluation of septicemia in children with Broviac catheters. J Pediatr 1984; 104: 29-33

119 Rijnders BJ, Wijngaerden E Van, Vandecasteele SJ et al. Treatment of long-term intravascular catheter-related bacteraemia with antibiotic lock: randomized, placebo-controlled trial. J Antimicrob Chemother 2005; 55: $90-94$

120 Robinson JL. Sensitivity of a blood culture drawn through a single lumen of a multilumen, long-term, indwelling, central venous catheter in pediatric oncology patients. J Pediatr Hematol Oncol 2002; 24: $72-74$

121 Rubin LG, Shih S, Shende A et al. Cure of implantable venous port-associated bloodstream infections in pediatric hematology-oncology patients without catheter removal. Clin Infect Dis 1999; 29: 102 - 105

122 Safdar N, Fine JP, Maki DG. Meta-analysis: methods for diagnosing intravascular device-related bloodstream infection. Ann Intern Med 2005; 142: 451 - 466

123 Salzer W, Steinberg SM, Liewehr DJ et al. Evaluation and treatment of fever in the non-neutropenic child with cancer. J Pediatr Hematol Oncol 2003; 25: 606-612

124 Salzman MB, Rubin LG. Intravenous catheter-related infections. Adv Pediatr Infect Dis 1995; 10: 337 - 368

125 Salzman MB, Rubin LG. Relevance of the catheter hub as a portal for microorganisms causing catheter-related bloodstream infections. Nutrition 1997; 13: 15S-17S

126 Seifert H, Cornely O, Seggewiss K et al. Bloodstream infection in neutropenic cancer patients related to short-term nontunnelled catheters determined by quantitative blood cultures, differential time to positivity, and molecular epidemiological typing with pulsed-field gel electrophoresis. J Clin Microbiol 2003; 41: 118-123
127 Shah CB, Mittelman MW, Costerton JW et al. Antimicrobial activity of a novel catheter lock solution. Antimicrob Agents Chemother 2002; 46: $1674-1679$

128 Shankar KR, Abernethy LJ, Das KS et al. Magnetic resonance venography in assessing venous patency after multiple venous catheters. J Pediatr Surg 2002; 37: 175 - 179

129 Shulman RJ, Phillips S, Laine L et al. Volume of blood required to obtain central venous catheter blood cultures in infants and children JPEN J Parenter Enteral Nutr 1993; 17: 177-179

130 Siegman-Igra Y, Anglim AM, Shapiro DE et al. Diagnosis of vascular catheter-related bloodstream infection: a meta-analysis. J Clin Microbiol 1997; 35: $928-936$

131 Simon A, Fleischhack G. [Surveillance for nosocomial infections in pediatric hematology/oncology patients]. Klin Padiatr 2001; 213 (Suppl 1): A $106-$ A 113

132 Simon A, Fleischhack G, Hasan C et al. Surveillance for nosocomial and central line-related infections among pediatric hematology-oncology patients. Infect Control Hosp Epidemiol 2000; 21: 592 - 596

133 Simon A, Gröger N, Engelhart S et al. Vancomycin-resistente Enterokokken [VRE]. Übersicht zu Bedeutung, Prävention und Management in der Pädiatrie. Hygiene und Medizin 2004; 29: 259-275

134 Simon A, Schaaf F, Marklein G et al. Methicillin-resistente Staphylococcus aureus [MRSA]. Übersicht zu Bedeutung und Management in der stationären Kinderheilkunde. Hygiene und Medizin 2003; 28: $62-74$

135 Singh-Naz N, Sleemi A, Pikis A et al. Vancomycin-resistant Enterococcus faecium colonization in children. J Clin Microbiol 1999; 37: 413 416

136 Sitges-Serra A, Girvent M. Catheter-related bloodstream infections. World J Surg 1999; 23: 589 - 595

137 Skladal D, Horak E, Maurer K et al. Complications of percutaneous insertion of Hickman catheters in children. J Pediatr Surg 1999; 34: $1510-1513$

138 Smith TL, Pullen GT, Crouse V et al. Bloodstream infections in pediatric oncology outpatients: a new healthcare systems challenge. Infect Control Hosp Epidemiol 2002; 23: 239 - 243

139 Sofka K, Wiszniewsky G, Blaser G et al. Antibakterieller Honig (Medihoney ${ }^{\mathrm{TM}}$ ) zur Wundpflege. Wundantisepsis bei pädiatrischen Patienten in der Hämatologie-Onkologie? Krankenhaushygiene und Infektionsverhütung 2004; 26: 183 - 187

140 Torres-Viera C, Thauvin-Eliopoulos C, Souli M et al. Activities of taurolidine in vitro and in experimental enterococcal endocarditis. Antimicrob Agents Chemother 2000; 44: 1720-1724

141 Traub WH, Leonhard B, Bauer D. Taurolidine: in vitro activity against multiple-antibiotic-resistant, nosocomially significant clinical isolates of Staphylococcus aureus, Enterococcus faecium, and diverse Enterobacteriaceae. Chemotherapy 1993; 39: 322 - 330

142 Trautmann M, Krier C. Katheterinfektionen - Prävention, Diagnose und Management von Infektionen durch intravasale Katheter. 1. Aufl. Thieme Verlag, Stuttgart 2004

143 Tunkel AR. Adjunctive Dexamethasone in Bacterial Meningitis in Children. Curr Infect Dis Rep 2003; 5: 319

144 Tunkel AR, Sepkowitz KA. Infections caused by viridans streptococci in patients with neutropenia. Clin Infect Dis 2002; 34: 1524-1529

145 Hoff J van, Berg AT, Seashore JH. The effect of right atrial catheters on infectious complications of chemotherapy in children. J Clin Oncol 1990; 8: 1255 - 1262

146 Viale P, Pagani L, Petrosillo N et al. Antibiotic lock-technique for the treatment of catheter-related bloodstream infections. J Chemother 2003; 15: $152-156$

147 Weisman LE. Coagulase-negative staphylococcal disease: emerging therapies for the neonatal and pediatric patient. Curr Opin Infect Dis 2004; 17: $237-241$

148 Zinner SH. Changing epidemiology of infections in patients with neutropenia and cancer: emphasis on gram-positive and resistant bacteria. Clin Infect Dis 1999; 29: 490-494 